\title{
The role of within-trial location of the retention interval in rats' delayed conditional discrimination performance
}

\author{
J. S. COHEN, J. GRASSI, and P. DOWSON \\ University of Windsor, Windsor, Ontario, Canada
}

\begin{abstract}
Rats were trained and tested on delayed conditional discriminations (DCDs) consisting of four possible light and tone stimulus sequences: light-light, tone-tone, light-tone, and tone-light. A lever was presented after the offset of the second or test stimulus, $\mathbf{S}_{2}$. Two retention intervals (RIs) were present within the DCD task, one (RI-1) between the sample or first stimulus $\left(\mathrm{S}_{1}\right)$ and $S_{2}$, and the other (RI-2) between $S_{2}$ and presentation of the lever. Liquid reinforcement was contingent upon pressing only when $S_{2}$ matched $S_{1}$ in Experiment 1 or only when $S_{2}$ differed from $S_{1}$ in Experiment 2. RI-1 and RI-2 were separately increased to 5, 10, and $20 \mathrm{sec}$ from 1-sec training conditions. Increasing RI-1 produced greater declines in performance to light $S_{1}$ than to tone $S_{1}$ in both experiments. No such stimulus modality effect occurred for increases in RI-2 in these experiments. These results indicate that retrospection of $\mathrm{S}_{1}$ occurred during RI-1 and prospection of a response decision and reward expectancy primarily occurred during RI-2.
\end{abstract}

Honig and Thompson (1982) distinguished between retrospection (memory of a prior event) and prospection (retention of instructions for future responding and expected outcomes) in nonhuman short-term memory. Variants of Konorski's (1959) successive delayed matchingto-sample (DMTS) paradigm have been used to test this dual-process model in pigeons. Wallace, Steinert, Scobie, and Spear (1980) adapted this paradigm for rats by using changes in global visual and auditory stimulation in the operant chamber. In successive DMTS tasks, temporal sequences of two stimuli are presented in four possible combinations: A-A, B-B, A-B, and B-A. In the original paradigm, reinforcement is contingent upon responding to the second, comparison stimulus, $S_{2}$, only when it matches the first, sample stimulus, $S_{1}(A-A, B-B)$, and not when it differs (A-B, B-A). Either true matching or symbolic matching (when comparison stimuli are different attributes of the same or of a different dimension from $S_{1}$ ) may be used. Also possible is a symmetrically reinforced version of this task in which responding in one way to matching pairs and in another way to mismatching pairs is rewarded (D'Amato \& Worsham, 1974; White, 1974). Another variant, the delayed sequence discrimination (DSD) problem, requires the organism to respond to only one specific temporal sequence (Weisman, Wasserman,

Support for this research came from Natural Sciences and Engineering Research Council of Canada Operating Grant A8249 and a University of Windsor research grant to the first author. Experiment 2 served as a thesis in partial fulfillment for the BA honors degree to the second author by the University of Windsor. Experiments 1 and 2 were initially presented at the Conference on Animal Learning, University of Toronto, June 1986. The authors thank N. Innis, University of Western Ontario, for reviewing the manuscript. Direct requests for reprints to J. S. Cohen, Department of Psychology, University of Windsor, Windsor, ON N9B 3P4, Canada.
Dodd, \& Larew, 1980). In all of these forms of the delayed conditional discrimination (DCD) task, retention of $S_{1}$ is assessed by increasing the interstimulus or retention interval (RI) between $S_{1}$ and $S_{2}$.

The basic method for investigating the dual-process model has been to vary the DMTS task so that the animal can use prospection, rather than retrospection of $S_{1}$, for a response. One way to accomplish this end is to change the DMTS task into a simple delayed discrimination (DD) problem; another is to relocate the RI from between $S_{1}$ and $S_{2}$ to between $S_{2}$ and the opportunity to respond. In the DD problem, $S_{1}$ now provides all the information about responding for reinforcement at $S_{2}$. When RI is shifted to follow $S_{2}$, the subject needs to prospect its decision about responding, rather than to retrospect the nature of $S_{1}$. As Honig and Wasserman (1981) pointed out, the successive DMTS paradigm is better suited to investigate the dual-process theory than is the simultaneous DMTS paradigm (Blough, 1959) in which the subject selects a matching test stimulus from two simultaneously presented test stimuli. Changing a successive DMTS task into a simple DD task will not alter stimulus sequences or the basic overall reinforcement schedule. Changing a simultaneous DMTS task into a DD problem, however, introduces a new reinforcement schedule if only half the trials contain the positive $S_{1}$ in the latter. Smith (1967) did maintain the same reinforcement schedule in both tasks by using a response-differentiated DD problem, but also used different sample and test stimuli in that task. It should also be noted that manipulation of RI location cannot be carried out in a simultaneous DMTS task.

The major finding with pigeons when successive DMTS tasks are changed to allow for response prospection is enhanced accuracy of performance. Honig and Wasserman (1981) found that pigeons' performance declined less on 
a DD than on a DMTS task when RI was increased. Increasing the RI following $S_{2}$ also disrupts pigeons' accuracy of performance less than does increasing the RI following $S_{1}$ in DMTS tasks (Honig \& Dodd, 1983; Pontecorvo, 1985) and in the DSD problem (Weisman \& DiFranco, 1981).

Unfortunately, differences in general accuracy of performance may not offer adequate evidence for dual processing in short-term memory. A unitary prospective process could account for such differences, as Honig and Wasserman (1981) suggest. Perhaps during the RI following $S_{1}$ in the DMTS task, the animal prospects an "if, then" rule (Roitblat, 1980, 1982), so that it must retain more information about the nature of the positive $S_{2}$, the necessary response, and the outcome than it must in a DD task or during the RI following $S_{2}$, where it need only remember how it should respond when the opportunity to do so occurs. Even if retrospection of $S_{1}$ occurs during the RI before $S_{2}$, there is no logical reason why this type of information should be more difficult to retain than a prospected response decision generated by $S_{2}$ in a DMTS task or by $S_{1}$ in a DD task.

Differences more subtle than those in general accuracy of performance are needed to provide evidence of dual processing of information. Pontecorvo (1985), for example, also found that sample stimuli from previous trials proactively interfered with matching to $S_{1}$ in current trials only when the RI prior to $S_{2}$ was increased. This findings is consistent with the notion that retrospection of $S_{1}$ from one trial may be influenced by any persistent retention from previous $S_{1}$ stimuli. More direct evidence of $S_{1}$ retrospection could be shown, however, if delayed matching were influenced by the physical characteristics of $S_{1}$. Any manipulation designed to promote response/reward prospection ought to attenuate such differences in $S_{1}$ matching. Urcuioli and Zentall (1986) demonstrated possible retrospection of $S_{1}$ by pigeons in simultaneous DMTS tasks. Their birds matched better to color than to line-orientation sample stimuli, regardless of the dimension of test stimuli. Evidence of $S_{\mathbf{1}}$ retrospection in rats has been shown by better delayed matching to onset of a tone than to onset of chamber lights in a successive DMTS task (Cohen, Escott, \& Ricciardi, 1984; Wallace et al., 1980, Experiment 2). In both studies, reinforcement was contingent upon leverpressing to a light or tone $S_{2}$ only when it matched a light or tone $S_{1}$. Cohen, Galgan, and Fuerst (1986) showed that this effect was based on the modality of $S_{1}$, not that of $S_{2}$, and, more importantly, that the effect either disappeared or was attenuated when the DMTS problem was changed into a DD task, even though general performance did not necessarily improve in the latter.

Cohen et al. (1986) demonstrated that changing a DMTS task into a DD task changed the way rats retain information for responding to $S_{2}$. The question posed in the present study is whether both processes, retrospection and prospection, are used in a temporally sequenced manner in the DMTS task. That is, do rats try to retain some representation of $S_{1}$ until $S_{2}$ (retrospection) and then retain their decision about responding until they have the opportunity to exercise that decision (prospection)? Such a temporal sequence of these processes has been suggested by investigators, already cited, who varied RI location. The present study, therefore, is an extension of previous research by Cohen et al. (1986). In the present research, we observed whether changing the location of increasing RI within a successive DMTS would influence the effect of the modality of the sample stimulus on delayed matching accuracy. On some tests for delayed responding, the interval between offset of the sample stimulus and onset of the test stimulus (RI-1) was increased; on other tests, the interval between offset of the test stimulus and presentation of a lever (RI-2) was increased. We expected that the effect of $S_{1}$ modality on accuracy of responding would be restricted to increases in RI-1; that is, matching would decline more to the light $S_{1}$ than to the tone $S_{1}$. The effect of $S_{1}$ modality was expected to disappear or diminish when only RI-2 was increased, because the rat would primarily be prospecting its decision to respond from $\mathrm{S}_{2}$, rather than retrospecting the nature of $S_{1}$. Two experiments were carried out in this study. In Experiment 1, a true matching DMTS task was used. Experiment 2 was similar to Experiment 1 except that a true delayed mismatching-to-sample (DMmTS) task was used. In this task, reinforcement was contingent upon responding to $S_{2}$ only when it differed from $S_{1}$ (light-tone, tone-light sequences), rather than when $S_{1}$ and $S_{2}$ matched, as in DMTS (light-light, tone-tone). The rationale for this variation is given in the introduction to Experiment 2.

\section{EXPERIMENT 1}

\section{Method}

\section{Subjects}

Six naive male albino rats (Wistar strain) bred in our colony served as subjects when they had reached 90-150 days of age. Each rat was individually housed and was provided with free access to dry food and restricted (20-30 $\mathrm{min}$ ) access to water, approximately $30 \mathrm{~min}$ following each daily experimental session. The animals experienced a 12:12-h light:dark cycle and were run during the dark cycle. Approximately every 2 weeks, the animals received free access to water for $48 \mathrm{~h}$ followed by restricted access to water over the next $24 \mathrm{~h}$. They were not run during this period. These breaks in the running schedule were employed to maintain the health of our subjects over the lengthy duration of the experiment. The only exception to this procedure occurred when an animal was on a block of retention test sessions, in which case its free access period was delayed until after its test sessions.

\section{Apparatus}

Four commercially available operant chambers (Tech Serv), described by Cohen et al. (1984), were used. Each chamber was enclosed in a light-and-sound-attenuating isolation chamber, which contained a ventilation fan that produced a constant masking noise of $55 \mathrm{~dB}$. An external speaker in the running room also emitted 62-dB white noise to further mask sounds from electromechanical programming and recording equipment in the adjacent room. Each chamber contained a retractable lever and a $.05-\mathrm{ml}$ water dipper on one wall and five 24-V 6-W incandescent lights and a Mallory 
Sonalert $2.8-\mathrm{kHz}$ tone generator in the ceiling. A $75-\mathrm{dB}$ tone was produced by this generator. Two stimulus events, either an onset of the ceiling lights or a $75-\mathrm{dB} 2.8-\mathrm{kHz}$ tone, were presented in each operant chamber, which otherwise remained dark during intertrial and retention intervals. The lever could be programmed for insertion at any time from the onset of the second stimulus event onward and for retraction upon being pressed or after $5 \mathrm{sec}$ had elapsed. The water dipper delivered a .05-ml droplet of sweetened water $(.10 \%$ sodium cyclamate, $w / v)$ as a reinforcement for a correct leverpress.

\section{Procedure}

Shaping and initial DMTS training, in which the lever was presented with onset of $S_{2}$, were as described by Cohen et al. (1984). The following description focuses on final training and testing, in which the lever was presented after termination of $S_{2}$.

Each rat first acquired the DMTS task in which the lever was presented with onset of $S_{2}$. Four sequential pairs of 2.5 -sec light and tone stimuli were presented: light-light, tone-tone, light-tone, and tone-light. Each pair appeared equally often over 100 trials in each session in a semirandom order so that matching or mismatching pairs or pairs with the same initial stimulus, $S_{1}$, did not occur more than three times in a row. During training, RI-1 was maintained at $1 \mathrm{sec}$. Intertrial intervals between successive stimulus pairs were $24 \mathrm{sec}$ from the offset of $S_{2}$. Reinforcement was contingent upon a leverpress to $S_{2}$ only if $S_{2}$ matched $S_{1}$. No reinforcement occurred for mismatching pairs. Thus, a maximum of 50 reinforcements, 25 to each matching pair, was possible within each session. Criterion for acquisition of this task was 30 correct presses out of 40 on each of two successive sessions. This criterion ensured that the rat could not achieve $75 \%$ correct leverpressing with a low number of presses.

Following acquisition of the initial DMTS task, each rat was trained to the same criterion with presentation of the lever delayed first $1 \mathrm{sec}$, then $2 \mathrm{sec}$, and finally $3.5 \mathrm{sec}$ from $S_{2}$ onset. The final delay allowed the lever to emerge $1 \mathrm{sec}$ after offset of $S_{2}(1 \mathrm{sec}$ RI-2). Intertrial intervals were now determined from presentation of the lever and onset of the next $S_{1}$ and were kept constant at $23 \mathrm{sec}$ throughout the remaining phases of the experiment.

Retention tests. Tests for $S_{1}$ retention were carried out by increasing either RI- 1 or RI-2 to 5,10 , and $20 \mathrm{sec}$ for each rat after it had reached criterion with both RIs at $1 \mathrm{sec}$. After reaching criterion on two successive sessions ( 200 trials) with $1-\sec$ RIs, the rat recieved a block of two sessions ( 200 trials) in which one of the RIs was increased to $5 \mathrm{sec}$ and the other remained at $1 \mathrm{sec}$. Three rats had RI-1 increased to $5 \mathrm{sec}$ while RI-2 remained at $1 \mathrm{sec}$, and three had RI-2 increased to $5 \mathrm{sec}$ while RI-1 remained at $1 \mathrm{sec}$. Following this test block, each rat was retrained to criterion with each RI at $1 \mathrm{sec}$. A second block of test trials over two sessions was then presented with the duration of the other RI increased to $5 \mathrm{sec}$ and the previously increased RI now at $1 \mathrm{sec}$. The same procedure was used for testing $S_{1}$ retention at RIs of 10 and $20 \mathrm{sec}$. Each test block always followed reacquisition sessions with both RIs at 1 sec. Each rat experienced six $S_{1}$ retention test blocks following reacquisition blocks. Three animals received their six retention test blocks in the following order: 5-sec RI-1, 5-sec RI-2, 10-sec RI-2, 10-sec RI-1, 20-sec RI-1, and 20-sec RI-2. The other three rats received the opposite alternating pattern of location of increased $\mathrm{RIs}$ in an ascending order.

Retraining each animal to criterion with both RIs at $1 \mathrm{sec}$ after each retention test block of sessions allowed it to recover from any effects of testing that might affect its responding on the next block of test sessions.

Data analysis. Each block of training or reacquisition sessions to criterion of 200 trials produced baseline performance before the next block of 200 retention test trials. Each baseline block and retention test block contained 100 matching and 100 mismatching pairs of stimuli, half beginning with the visual $S_{1}$ and half beginning with the auditory $S_{1}$. Proportions of leverpresses out of 50 possible presses to each stimulus pair at each baseline and retention block were calculated. Proportions of reinforced presses, or hits, and nonreinforced presses, or false alarms, were used to calculate $A^{\prime}$, a retention index for $S_{1}$ at each criterion and test block of sessions.

$A^{\prime}$ is a nonparametric measure of signal sensitivity from signal detection theory (McNicol, 1972; Pollack, 1970) that may be used to determine retention of an event. We used this measure instead of a discrimination ratio because $A^{\prime}$ attenuates the bias to make positive responses that is inherent in successive DMTS tasks, according to Weiskrantz (1968). A more complete justification for using $A^{\prime}$ and its mathematical derivation is presented in earlier reports from this laboratory (Cohen et al., 1984; Cohen et al., 1986) and in more general treatments of signal detection theory (McNicol, 1971 ; Pollack, 1970). $A^{\prime}$ is calculated algebraically (Pollack, 1970) by the following formula:

$$
A^{\prime}=[(y-x)(1.00+y-x) / 4 y(1.00-x)]+0.5,
$$

where $y=$ proportion of hits and $x=$ proportion of false alarms. $A^{\prime}$ scores normally range from .50 , indicating no signal sensitivity or retention because $x=y$, to 1.00 , indicating perfect signal sensitivity or retention because $y=1.00$ and $x=0 . A^{\prime}$ of less than .50 is possible when $y<x$, but such differences between hits and false alarms seldom consistently occur in our preparation.

Retention of the visual $S_{1}$ was estimated from $A^{\prime}$ scores based on proportions of hits to the light-light pairs and of false alarms to the light-tone pairs. Retention of the auditory $S_{1}$ was estimated from $A^{\prime}$ scores based on proportions of hits to tone-tone pairs and of false alarms to tone-light pairs. Two sets of $A^{\prime}$ scores were calculated, one for increases in duration of RI- 1 and the other for increases in duration of RI-2. $A^{\prime}$ scores were calculated from RIs of $1 \mathrm{sec}$ at each location during reacquisition blocks of sessions preceding each retention test block containing a longer RI at one of the locations.

Individual $A^{\prime}$ scores were used to calculate mean $A^{\prime}$ scores to construct figures in this study, but were not directly used for parametric statistical analysis because they are proportion measures. Therefore, individual $A^{\prime}$ scores were transformed into arcsin scores to normalize their binomial distributions and stabilize variances, as suggested by Winer (1971). Arcsin scores were subjected to a three-way analysis of variance (ANOVA) $\left(S_{1}\right.$ modality [light, tone] $\times$ RI location [RI-1, RI-2] $\times$ RI duration $[1,5,10$, or $20 \mathrm{sec}])$ with repeated measures on all factors. Although data were gathered from training sessions with RI-1 and RI-2 both at 1 sec prior to each block of retention test sessions, only data from the training sessions before the retention test block with RI-1 at $5 \sec$ (RI-2 at $1 \mathrm{sec}$ ) and before the retention test block with RI-2 at $5 \mathrm{sec}$ (RI-1 at $1 \mathrm{sec}$ ) were used in this analysis. Subsequent baseline data were ignored to simplify the ANOVA; this was justified by the fact that baseline $A^{\prime}$ scores did not differ significantly over training blocks, as established by a separate three-way ANOVA for 1-sec RIs in each experiment. Newman-Keuls tests were used for individual comparisons.

\section{Results and Discussion}

Figure 1 shows mean $A^{\prime}$ scores for each $S_{1}$ at each $R I$ location and duration in the first experiment. Examination of this figure reveals that a sample-stimulus modality effect was restricted to increases in RI-1. $A^{\prime}$ declined more to the light $S_{1}$ than to the tone $S_{1}$ only with increases in RI-1. Increases in RI-2 led to similar declines in $A^{\prime}$ to either $S_{1}$. Location of increasing RI primarily affected accuracy of matching to the light $S_{1} . A^{\prime}$ to the light $S_{1}$ declined more with increases in RI-1 than with increases 


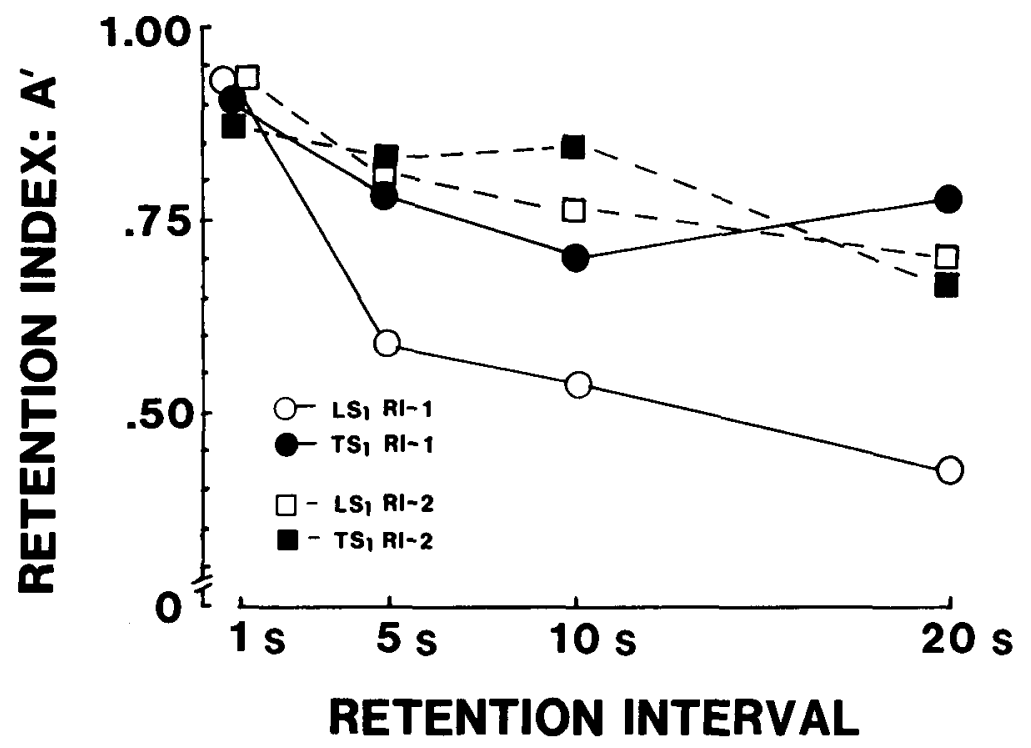

\begin{abstract}
Figure 1. Mean $A^{\prime}$ retention scores for delayed matching to each sample stimulus for RI-1 and RI-2 at 1-, 5-, 10-, and 20-sec durations in Experiment 1. Only data from 1-sec RIs preceding testing with 5-sec RIs are shown. $\mathbf{L S}_{\mathbf{1}}=$ light sample stimulus, $\mathbf{T S}_{\mathbf{1}}=$ tone sample stimulus.
\end{abstract}

in RI-2. Increasing RI-1 or RI-2 to $20 \mathrm{sec}$ produced greater declines in $A^{\prime}$ to the light $\mathrm{S}_{1}$ than did increasing either RI to 5 or $10 \mathrm{sec}$. A similar retention function was also seen for declines in $A^{\prime}$ to the tone $S_{1}$ for greater increases in RI-2. A significant interaction among all three factors $[F(3,15)=5.92, p<.01]$ and individual comparisons $(p<.05)$ supported these observations. $A^{\prime}$ to tone $S_{1}$ also declined more for an increase to $10 \mathrm{sec}$ at RI-1 than at RI-2, but declined less for an increase to $20 \mathrm{sec}$ at RI-2 than at RI-1.

The results of this experiment are consistent with a dualprocess interpretation that retrospection of the nature of $\mathrm{S}_{\mathbf{1}}$ during RI-1 is taken over by prospection of a specific response decision during RI-2. This view is supported by the fact that modality of $S_{1}$ influenced delayed performance only for increases in RI-1. It should also be noted that possible prospection during RI-2 was not always better than possible retrospection during RI-1. Matching to the tone $S_{1}$ did not consistently decline more for increases in RI-1 than for increases in RI-2, as was found for matching to the light $S_{1}$.

Our results, however, could be accounted for by a single prospective process similar to one proposed by Roitblat $(1980,1982)$. Each sample stimulus may elicit in the subject a specific "if, then" type of prospective representation, allowing it to anticipate what test stimulus requires a response for reinforcement. Perhaps the rat finds anticipating responding to a possible light $S_{2}$ more difficult to rehearse or retain than anticipating responding to a possible tone $S_{2}$ as elicited by a specific $S_{1}$. Therefore, when $S_{2}$ is presented and the correct prospection to respond to an expected light $S_{2}$ is not well remembered, the rat may respond anyway to ensure hits, even though false alarms will also increase. If the correct prospection is to respond to a tone $S_{2}$ that is better remembered, the rat will be better able to compare the actual $S_{2}$ with its expected $S_{2}$, both to maintain hits and to prevent false alarms. Unfortunately, results from a DMTS task alone cannot determine whether the $S_{1}$ modality effect results from differences in retrospection of $S_{1}$ or in prospection for $S_{2}$. Comparisons between the results of a DMTS task and a mismatching version of this task can determine which process prevails during each RI. Experiment 2 was run to answer this question.

\section{EXPERIMENT 2}

According to the prospection hypothesis of the modality effect discussed above, delayed conditional discrimination should be poorer for any sample stimulus that elicits a prospection for a possible light $S_{2}$ than for a tone $S_{2}$. The modality of $S_{1}$, therefore, should not matter; only the modality of the expected $S_{2}$ should account for any modality effects. To test this hypothesis, we trained rats on a DMmTS task in which reinforcement was contingent upon leverpressing only when $S_{2}$ differed from $S_{1}$ (light-tone, tone-light pairs). The prospection hypothesis would predict that now increases in RI-1 should reduce accuracy of performance more to the tone $S_{1}$, which elicits the harder-to-remember prospection to respond if $S_{2}$ is light, than to the light $S_{1}$, which elicits an easier-toremember prospection to respond if $S_{2}$ is tone. According to the retrospection hypothesis, mismatching will decline more to the light $S_{1}$ than to the tone $S_{1}$. Once the animal decides what to do at $S_{2}$, however, prospection of this response decision that is no longer associated with modality of either $S_{1}$ or the anticipated $S_{2}$ will take over. Therefore, according to either hypothesis, increasing RI-2 
with RI-1 maintained at $1 \mathrm{sec}$ should result in declines in accuracy that are unaffected by stimulus modality.

\section{Method}

\section{Subjects and Procedure}

Seven experimentally naive male albino rats were trained and tested in a DMmTS task. Procedures identical to those used for training, testing, and data analysis in the first experiment were employed. Only contingencies for reinforcement differed in this ex periment, in that reinforcement was contingent for leverpressing when $S_{2}$ differed from $S_{1}$ (light-tone, tone-light). No reinforcement was available for matching stimulus pairs (light-light, tonetone). $A^{\prime}$ for the light $S_{1}$ was calculated from hits to light-tone pairs and false alarms to light-light pairs. $A^{\prime}$ for the tone $S_{1}$ was calculated from hits to tone-light pairs and false alarms to tone-tone pairs.

\section{Results and Discussion}

Figure 2 shows mean $A^{\prime}$ scores to each $S_{1}$ at each RI duration and location in Experiment 2. A comparison of Figures 1 and 2 reveals highly similar functions. Only increasing RI-1 resulted in greater reductions of $A^{\prime}$ to the light $S_{1}$ than to the tone $S_{1}$ in Experiment 2. As in the first experiment, location of increasing RI affected $A^{\prime}$ only to the light $S_{1} . A^{\prime}$ to the light $S_{1}$ declined more for increases in RI-1 than in RI-2. Declines in $A^{\prime}$ were more generally directly related to RI duration in this experiment. Declines in $A^{\prime}$ to either $S_{1}$ were greater for an increase in either RI to $20 \mathrm{sec}$. Confirmation of these observations was provided by a significant interaction among all three variables $[F(3,18)=11.95, p<.01]$ and by individual comparisons $(p<.05)$.

The results of Experiment 2 are also consistent with the notion that retrospection of $S_{1}$ occurs during RI-1 and prospection of a response decision occurs during RI-2. The stimulus-modality effect that occurred only for in- creasing RI-1 was only a function of the modality of $S_{1}$, which further supports the notion that this phenomenon indicates some form of $S_{1}$ retrospection during RI-1. The results of this experiment replicate those obtained by Cohen et al. (1986, Experiment 3) and are consistent with those reported by Urcuioli and Zentall (1986, Experiment 2). This experiment extends Cohen et al.'s (1986) findings, however, by showing that the effect of $S_{1}$ modality does not carry over to increases in post- $S_{2}$ delays. As in Experiment 1, location of RI increases affected accuracy of delayed responding to the light $S_{1}$ but not to the tone $S_{1}$.

\section{GENERAL DISCUSSION}

Temporal sequencing of retrospective and prospective processes in the rat's working memory was shown by observing the influence of modality of a sample stimulus on delayed performance as a function of location of RI increases in two DCD tasks, successive DMTS and DMmTS problems. Retrospection, as indicated by poorer accuracy of conditional responding to a visual than to an auditory sample stimulus, was restricted to increases in the RI between sample and test stimuli in both experiments. The notion that the stimulus-modality effect is an indicant of $S_{1}$ retrospection, rather than $S_{2}$ prospection, was supported by the fact that only the modality of the sample stimulus was responsible for differences in delayed responding. These results are consistent with Honig and Thompson's (1982) dual-process model of working memory, and extend earlier work in our laboratory (Cohen et al., 1986) that showed that the stimulusmodality effect is either eliminated or greatly attenuated by transforming a DMTS task into a DD task.

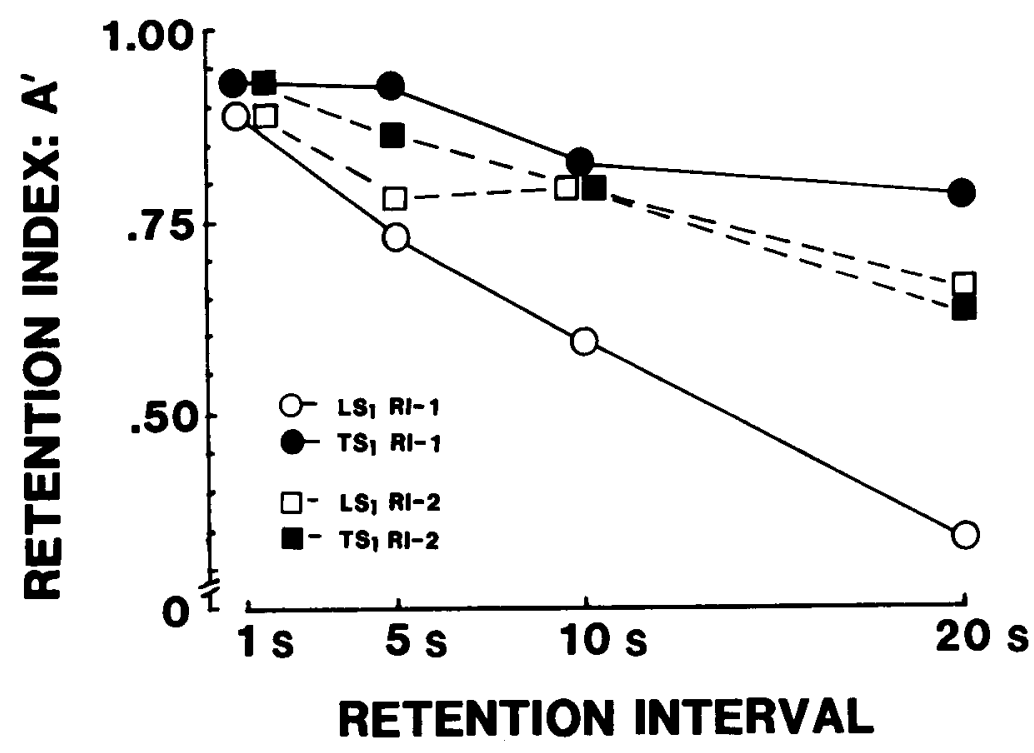

Figure 2. Mean $A^{\prime}$ retention scores for delayed mismatching to each sample stimulus for RI-1 and RI-2 at 1-, 5-, 10, and 20-sec durations in Experiment 2. Only data from 1-sec RIs preceding testing with 5-sec RIs are shown. $\mathbf{L S}_{\mathbf{1}}=$ light sample stimulus, $\mathrm{TS}_{\mathbf{1}}=$ tone sample stimulus. 
The present findings provide further and more conclusive evidence that in a DMTS task the animal retrospects the nature of $S_{1}$ until it receives $S_{2}$, at which time it decides what to do and then prospects its decision and expectancy for reward. That these processes need not be differentiated on the basis of general accuracy of performance was also demonstrated in this study. Our experiments provide better support for the temporal sequencing model than have previous studies in another way, as well. We demonstrated the effect of increasing RI at different locations through manipulating this variable for the same rats in the same basic DCD task in an alternating manner. Both Honig and Dodd (1983) and Pontecorvo (1983) inferred such a sequence of processes from separate groups of pigeons for whom duration of only one or the other RI was varied. Furthermore, Honig and Dodd's Conditional Delayed Discrimination (CDD) task differed from their DCD task in that the sample and task stimuli occurred simultaneously in the former but successively in the latter. Weisman and DiFranco (1981) did maintain an interstimulus interval, as distinct from the posttest stimulus RI, and did use the same pigeons in comparing the effects of increasing each RI separately. However, they trained and tested their subjects with increased RIs first at one location and then at the other, rather than alternating test locations as we did.

Sequential sharing of different ways of processing in working memory may not be restricted to DCD tasks. A similar sequencing order of retrospection and prospection was proposed by Cook, Brown, and Riley (1985), who tested rats' performance in a 12 -arm radial maze. They found that 15-min delays disrupted choice accuracy less when interpolated during the early or late part of the choice sequence than when interpolated midway, after the sixth correct choice. Cook et al. (1985) suggested that rats, when making a choice, initially attempt to remember which arms they have previously sampled (retrospection), but as this memory load increases, they switch to trying to determine which arms have yet to be approached (prospection). In both preparations, retrospection occurs first and is supplanted by prospection, but in the radial arm maze the mechanism of a fixed capacity in working memory determines when the switch is made. In our DCD tasks, information about the likelihood of reinforcement for a response signaled by a stimulus determines whether retrospection or prospection is used. If $S_{1}$ offers no specific information about reinforcement until $S_{2}$ is presented, retrospection of a representation of $S_{1}$ must occur until it can be compared to $S_{2}$, at which time a prospection for a specific response and outcome expectancy can be formed.

Although temporal sequencing of dual processing was shown in this study, we cannot rule out the possibility of some form of prospection during RI-1. Pontecorvo (1985) did find that earlier responding, as well as earlier sample stimuli, proactively interfered with delayed matching in subsequent trials with increased RI-1. A strict temporal separation of the processes would have produced only proactive interference from earlier responding during RI-2 and only proactive interference from earlier sample stimuli during RI-1. The findings of Santi and Roberts (1985) also suggest that pigeons do form an "if, then" rule of prospective coding during RI-1. Santi and Roberts varied memory load on such prospective information in a simultaneous symbolic DMTS task by assigning either many comparison stimuli to be correct for each sample stimulus (many to one) or only one comparison stimulus to be correct for many sample stimuli (one to many). Performance declined more for the greater load (many to one) than for the lighter load (one to many) with increased RI-1. Retrospection of $S_{1}$, however, may have continued in either condition, since delayed matching appeared to be more accurate when sample and test stimuli were colors than when sample stimuli were line tilts or shapes. Therefore, we can conclude from our findings that retrospection of $S_{1}$ occurs during RI-1 and not during RI-2, but not that some form of prospection for $S_{2}$ is absent during RI-1. We can with more certainty, however, maintain that retrospection of $S_{1}$ is absent during RI-2, during which prospection of a response decision occurs.

\section{REFERENCES}

BLough, D. S. (1959). Delayed matching in the pigeon. Journal of the Experimental Analysis of Behavior, 2, 151-160.

Cohen, J. S., EscotT, M., \& Ricciardi, P. (1984). The role of reinforcement symmetry and stimulus modality in successive delayed matching to sample in the rat. Canadian Journal of Psychology, 38, 63-69.

Cohen, J. S., Galgan, R., Fuerst, D. (1986). Retrospective and prospective short-term memory in delayed response tasks in rats. Animal Learning \& Behavior, 14, 38-50.

COOK, R. G., Brown, M. F., \& RILEY, D. A. (1985). Flexible memory processing by rats: Use of prospective and retrospective information in the radial maze. Journal of Experimental Psychology: Animal Behavior Processes, 11, 453-469.

D'Amato, M. R., \& Worsham, R. W. (1974). Retrieval cues and shortterm memory in Capuchin monkeys. Joumal of Comparative \& Physiological Psychology, 86, 274-282.

HonIG, W. K., \& DodD, P. W. D. (1983). Delayed discrimination in the pigeon: The role of within-trial location of conditional cues. Animal Learning \& Behavior, 11, 1-9.

Honig, W. K., \& Thompson, R. K. R. (1982). Retrospective and prospective processing in animal working memory. In G. H. Bower (Ed.), The psychology of learning and motivation (Vol. 16, pp. 167197). New York: Academic Press.

Honig, W. K., \& Wasserman, E. A. (1981). Performance of pigeons on delayed simple and conditional discriminations under equivalent training conditions. Learning \& Motivation, 12, 149-170.

KoNORSKI, J. (1959). A new method of physiological investigation of recent memory in animals. Bulletin de L'Academie Polonaise des Sciences, 7, 115-119.

McNicol, D. (1972). A primer of signal detection theory. London: George Allen \& Unwin.

PollaCK, I. (1970). A nonparametric procedure for evaluations of true and false positives. Behavior Research Methods \& Instrumentation, 2, 155-156.

PonteConvo, M. J. (1985). Memory for a stimulus vs. anticipation of a response: Contrasting effects of proactive interference in two delayed comparison tasks. Animal Learning \& Behavior, 13, 355-364.

RoItBLAT, H. L. (1980). Codes and coding processes in pigeon shortterm memory. Animal Learning \& Behavior, 8, 341-351. 
Roitblat, H. L. (1982). The meaning of representation in animal memory. Behavioral \& Brain Sciences, 5, 353-406.

SAnti, A., \& Roberts, W. A. (1985). Prospective representation: The effects of varied mapping of sample stimuli to comparison stimuli and differential trial outcomes of pigeons' working memory. Animal Learming \& Behavior, 13, 103-108.

SMITH, L. (1967). Delayed discrimination and delayed matching in pigeons. Journal of the Experimental Analysis of Behavior, 10, 529-533.

UrCuloli, P. J., \& Zentall, T. R. (1986). Retrospective coding in pigeons' delayed matching-to-sample. Journal of Experimental Psychology: Animal Behavior Processes, 12, 69-77.

Wallace, J., Steinert, P. A., Scobie, S. R., \& Spear, N. E. (1980). Stimulus modality and short-term memory in rats. Animal Learning \& Behavior, 8, 10-16.

Weiskrantz, L. (1968). Memory. In L. Weiskrantz (Ed.), Analysis of behavioral change (pp. 158-188). New York: Harper \& Row.
Weisman, R. G., \& DiFranco, M. P. (1981). Testing models of delayed sequence discrimination in pigeons: Delay intervals and stimulus durations. Journal of Experimental Psychology: Animal Behavior Processes, 7, 413-424.

Weisman, R. G., Wasserman, E. A., Dodd, P. W. D., \& Larew, M. B. (1980). Representation and retention of two event sequences in pigeons. Journal of Experimental Psychology: Animal Behavior Processes, 6, 312-325.

WHITE, K. G. (1974). Temporal integration in the pigeon. British Journal of Psychology, 65, 437-444.

WINER, B. J. (1971). Statistical principles in experimental design (2nd ed.). New York: McGraw-Hill.

(Manuscript received April 27, 1987;

revision accepted for publication September 22, 1987.) 